\title{
FINITE DIFFERENCE METHODS FOR COMPUTING EIGENVALUES OF FOURTH ORDER BOUNDARY VALUE PROBLEMS
}

\section{RIAZ A. USMANI}

Pepartment of Applied Mathematics

University of Manitoba

Winnipeg, Manitoba, Canada

R3T 2N2

(Received Sepiember 7, 1984)

ABSTRACT. This brief report describes some new finite difference methods of order 2 and 4 for computing eigenvalues of a two point boundary value problem associated with a fourth order linear differential equation $y^{(4)}+(1(x)-\lambda q(x)) y=0$. These methods are derived from the formula

$$
\mathrm{h}^{4} \mathrm{y}_{1}^{(4)}=\left(\delta^{4}-\frac{1}{6} \delta^{6}+\frac{7}{240} \delta^{8}-\ldots\right) \mathrm{y}_{\mathrm{i}} \text {. }
$$

Numerical results are included to demonstrate practical usefulness of our methods.

KEY WORDS AND PHRASES. Central difference formula, Finite difference methods, Generalized symmetric eigenvalue problem, Positive definite matrix, Two-point boundary value problem. 1980 AMS SUBJECT CLASSIFICATION CODE: $65 L 15$.

\section{INTRODUCTION}

We shall consider the fourth order linear differential equation

$$
y^{(4)}+[p(x)-\lambda q(x)] y=0
$$

associated with one of the following pairs of homogeneous boundary conditions:

$$
\begin{aligned}
& \text { (a) } y(\text { a })=y^{\prime}(a)=y(b)=y^{\prime}(b)=0 \\
& \text { (b) } y(a)=y^{\prime \prime}(a)=y(b)=y^{\prime \prime}(b)=0 \\
& \text { (c) } y(\text { a })=y^{\prime}(a)=y^{\prime \prime}(b)=y^{\prime \prime}(\text { b })=0
\end{aligned}
$$

Such boundary value problems occur frequently in applied mathematics, engineering and modern physics, see $[1,2,3]$. In $(1.1)$, the functions $p(x), q(x) \in C[a, b]$ and they satisfy the conditions

$$
p(x) \geq 0, q(x)>0, x \in[a, b] .
$$

Recently, Chawla and Katti [4] have developed a finite difference method of order 2 for computing approximate values of $\lambda$ for a boundary value problem (1.1)-(1.2a). For the same problem, a fourth order method was developed by Chawla [5] which leads to a generalized seven-band symmetric matrix eigenvalue problem.

The purpose of this note is to present some new finite difference methods for computing approximate values of $\lambda$ for the boundary value problems (1.1)-(1.2b) and $(1.1)-(1.2 c)$. These methods lead to generalized five-band and seven-band symmetric matrix eigenvalue problem and provide $0\left(h^{2}\right)$ and $0\left(h^{4}\right)$-convergent 
approximations for the eigenvalues.

2. A SECOND ORDER METHOD FOR COMPUTING $\lambda$ FOR $(1.1)$ and $(1.2 \mathrm{~b})$

For a positive integer $N \geq 4$, let $h=(b-a) /(N+1)$ and $x_{i}=a+i h$, $i=0(1) N+1$. We shall designate $y_{i}=y\left(x_{i}\right), p_{i}=p\left(x_{i}\right)$ and $q_{i}=q\left(x_{i}\right)$. The boundary value problem $(1.1)$ and $(1.2 b)$ is discretized by the difference equations

$$
\begin{aligned}
& \text { (a) }-2 y_{0}+5 y_{1}-4 y_{2}+y_{3}=-h^{2} y_{0}^{\prime \prime}+h^{4}\left[-\frac{1}{12} y^{(4)}+y_{1}^{(4)}\right]+t_{1}, \\
& \text { (b) } \delta^{4} y_{i}=h^{4} y_{1}^{(4)}+t_{i}, i=2(1) N-1, \\
& \text { (c) } y_{N-2}-4 y_{N-1}+5 y_{N}-2 y_{N+1}=-h^{2} y_{N+1}^{\prime \prime}+h^{4}\left[y_{N}^{(4)}-\frac{1}{12} y_{N+1}^{(4)}\right]+t_{N} .
\end{aligned}
$$

Note that the truncation errors $t_{i}, i=1(1) \mathrm{N}$, are

$$
t_{i}=\left\{\begin{array}{l}
\frac{59}{360} h^{6}{ }^{(6)}\left(\xi_{1}\right), \xi_{1} \in\left(a, x_{3}\right) \\
\frac{1}{6} h^{6} y^{(6)}\left(\xi_{i}\right), \xi_{i} \in\left(x_{i-2}, x_{i}+2\right), i=2(1) N-1 \\
\frac{59}{360} h^{6} y^{(6)}\left(\xi_{N}\right), \xi_{N} \in\left(x_{N-2}, b\right) .
\end{array}\right.
$$

The formula $2.1(b)$ is obtained from the well-known central difference formula

$$
\begin{aligned}
h^{4} y_{i}^{(4)}= & {\left[\delta^{4}-\frac{1}{6} \delta^{6}+\frac{7}{240} \delta^{8}-\frac{41}{7560} \delta^{8}+\ldots\right] y_{i}, } \\
& (i=2,3,4, \ldots) .
\end{aligned}
$$

The discretizations $2.1(a)$ and 2.1 (c) are introduced so that the resulting coefficient matrix in (2.1) is a five-band symmetric matrix. The system of linear equations (2.1) can be written in matrix form

$$
\left(J^{2}+h^{4} P\right) Y=\lambda h^{4} Q Y+t,
$$

where $\mathrm{J}^{2}$ is a symmetric five-band matrix and $\mathrm{J}=\left(\mathrm{j}_{\mathrm{rs}}\right)$ is a tridiagonal matrix such that

$$
J_{r s}=\left\{\begin{array}{cl}
2, & r=s \\
-1, & |r-s|=1 \\
0, & \text { otherwise. }
\end{array}\right.
$$

The matrices $P$ and $Q$ are diagonal matrices

$$
P=\operatorname{diag}\left[\begin{array}{llll}
p_{1} & p_{2} & \cdots & p_{N}
\end{array}\right], Q=\operatorname{diag}\left[q_{1} q_{2} \cdot \cdots q_{N}\right]
$$

and

$$
Y=\left[\begin{array}{lll}
y_{1} & y_{2} \ldots y_{N}
\end{array}\right]^{T}, t=\left[t_{1} t_{2} \cdot t_{N}\right]^{T} .
$$

Thus, our method for computing approximations $\Lambda$ for $\lambda$ of (1.1)-(1.2b) can be expressed as a generalized five-band symmetric matrix eigenvalue problem

$$
\left(\mathrm{J}^{2}+\mathrm{h}^{4} \mathrm{P}\right) \tilde{\mathrm{Y}}=\Lambda \mathrm{h}^{4} \mathrm{Q} \tilde{Y} .
$$

In fact, the matrix $\mathrm{J}^{2}$ is a positive definite matrix and hence for any step-size $h>0$, the approximations $\Lambda$ for $\lambda$ by (2.6) are real and positive for all $p(x) \geq 0$ and $q(x)>0$. That our method provides $0\left(h^{2}\right)$ convervent approximations $\Lambda$ for $\lambda$ can be established following Grigorieft [6]. We omit the details of 
convergence proof for brevity.

3. A FOURTH ORDER METHOD

The boundary value problem $(1.1)-(1.2 b)$ is now discretized by the following difference equations

$$
\begin{aligned}
& \text { (a) }-17 y_{0}+44 y_{1}-38 y_{2}+12 y_{3}-y_{4}=-8 h^{2} y_{0}^{\prime \prime} h^{4}\left[\frac{1}{3} y_{0}^{(4)}+6 y_{1}^{(4)}\right]+\frac{13}{90} h^{6} y_{0}^{(6)}+\ldots \\
& \text { (b) } \quad 10 y_{0}-38 y_{1}+56 y_{2}-39 y_{3}+12 y_{4}-y_{5}=h^{2} y_{0}^{\prime \prime} \\
& +h^{4}\left[\frac{1}{12} y_{0}^{(4)}+6 y_{2}^{(4)}\right]+\frac{1}{360} h^{6} y_{0}^{(())}+\ldots \\
& \text { (c) }\left(6 \delta^{4}-\delta^{6}\right) y_{i}=6 h^{4} y_{i}^{(4)}-\frac{7}{40} h^{8} y_{i}^{(8)}+\ldots, i=3(1) N-2 \text {; } \\
& \text { (d) }-\mathrm{y}_{\mathrm{N}-4}+12 \mathrm{y}_{\mathrm{N}-3}-39 \mathrm{y}_{\mathrm{N}-2}+56 \mathrm{y}_{\mathrm{N}-1}-38 \mathrm{y}_{\mathrm{N}}+10 \mathrm{y}_{\mathrm{N}+1} \\
& =h^{2} y_{N+1}^{\prime \prime}+h^{4}\left[6 y_{N-1}^{(4)}+\frac{1}{12} y_{N+1}^{(4)}\right]+\frac{1}{360} h^{6} y_{N}^{(6)}+\ldots \text {, } \\
& \text { (e) }-y_{N-3}+12 y_{N}-2-38 y_{N-1}+44 y_{N}-17 y_{N}+1=-8 h^{2} y_{N}^{\prime \prime}+1 \\
& +h^{4}\left[6 v_{N}^{(4)}+\frac{1}{3} y_{N+1}^{(4)}\right]+\frac{13}{90} h^{6} y_{N}^{(6)}+\ldots
\end{aligned}
$$

As in [7], the derivation of (3.1c) is immediate from (2.3), on truncating the infinite series on the right of equality sign after the two terms. The additional difference equations $(3.1 \mathrm{a}, \mathrm{b}, \mathrm{d}, \mathrm{e})$ are chosen so that the resulting matrix associated with the system of linear equations in (3.1) is a seven-band symmetric matrix. It turns out that our method for computing approximations $\Lambda$ for $\lambda$ of (1.1)-(1.2b) can be expressed as a generalised seven-band symmetric matrix eigenvalue problem

$$
\left[\left(6 J^{2}+J^{3}\right)+6 h^{4} P\right] \tilde{Y}=6 \Lambda h^{4} Q \tilde{Y} .
$$

The matrix $6 \mathrm{~J}^{2}+\mathrm{J}^{3}$ is a positive definite matrix and hence for any step-size $\mathrm{h}>0$, the approximations $\Lambda$ for $\lambda$ by (3.2) are real and positive for all $p(x) \geq 0$ and $q(x)>0$. As before, it follows from the results of Grigorieff (1275) that our present method provides $O\left(h^{4}\right)$-convergent approximations $\Lambda$ for $\lambda$.

4. METHODS FOR COMPUTING $\lambda$ FOR $(1.1)-(1.2 \mathrm{c})$

For this section, let $h=(b-a) / N$ and $x_{i}=a+i h, i=0(1) N$. The boundary value problem $(1.1)-(1.2 c)$ is discretized by the following scheme

(a) $-4 y_{0}+7 y_{1}-4 y_{2}+y_{3}=2 h y_{0}^{\prime}+h^{4} y_{1}^{(4)}+\left(\frac{h^{3}}{3} y_{0}^{\prime \prime \prime}+\ldots\right)$,

(b) $\delta y^{4} y_{i}=h^{4} y_{i}^{(4)}+\frac{1}{6} h^{4} y^{(6)}\left(\xi_{i}\right), \xi_{i} \in\left(x_{i-2}, x_{i+2}\right)$,

$\mathrm{i}=2(1) \mathrm{N}-2$,

(c) $y_{N}-3-4 y_{N}-2+5 y_{N-1}-2 y_{N}=-h^{2} y_{N}^{\prime \prime}+\frac{11}{12} h^{4} y_{N-1}^{(4)}$

$$
+\left(\frac{1}{12} h^{5} y_{N}^{(5)}+\ldots\right) \text {, }
$$

(d) $y_{N-2}-2 y_{N-1}+y_{N}=h^{2} y_{N}^{\prime \prime}-h^{3} y_{N}^{\prime \prime}+\frac{7}{12} h^{4} y_{N}^{(4)}+\left(\frac{1}{4} h^{5} y_{N}^{(5)}+\ldots\right)$.

This system can be written in matrix form 


$$
\left(A+h^{4} P\right) y=\lambda h^{4} Q y+t
$$

and our method for computing approximation $\Lambda$ for $\lambda$ of (1.1)-(1.2c) can be written as a generalized five-band symmetric matrix eigenvalue problem

$$
\left(A+h^{4} P\right) \tilde{y}=\Lambda h^{4} Q \tilde{y}
$$

where

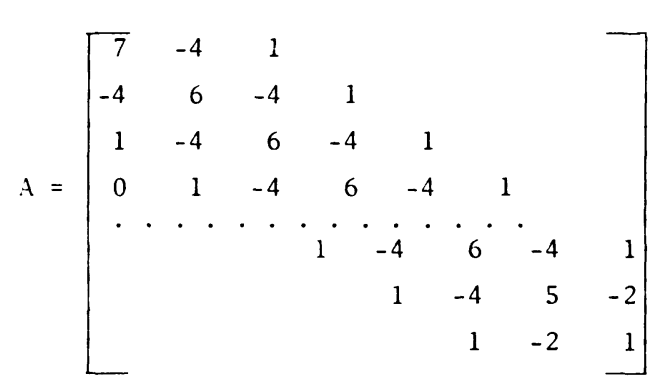

It can be established (see Appendix A) that the matrix $A$ is a positive definite matrix and the approximations $\Lambda$ for $\lambda$ by (4.3) are $0\left(h^{2}\right)$-convergent.

A third order method is obtained on discretizing (1.1)-(1.2c) by the difference equations

$$
\begin{aligned}
& \text { (a) }-45 y_{0}+76 y_{1}-42 y_{2}+12 y_{3}-y_{4}=24 h y_{0}^{\prime} \\
& +h^{4}\left(-y_{0}^{(4)}+6 y_{1}^{(4)}\right)-\frac{4}{5} h^{5} y_{0}^{(5)}-\left(\frac{1}{6} h^{6} y_{0}^{(6)}+\ldots\right), \\
& \text { (b) } \frac{27}{2} y_{0}-42 y_{1}+\frac{113}{2} y_{2}-39 y_{3}+12 y_{4}-y_{5}=-3 h y_{0}^{\prime} \\
& +h^{4}\left(\frac{1}{4} y_{0}^{(4)}+6 y_{2}^{(4)}\right)+\frac{1}{10} h^{5} y_{0}^{(5)}+\left(\frac{1}{24} h^{6} y_{0}^{(6)}+\ldots\right), \\
& \text { (c) }\left(6 \delta^{4}-\delta^{6}\right) y_{i}=6 h^{4} y_{i}^{(4)}-\left(\frac{7}{40} h^{8} y_{i}^{(8)}+\ldots\right), i=3(1) \mathrm{N}-3 \text {, } \\
& \text { (d) }-y_{N-5}+12 y_{N-4}-39 y_{N-3}+54 y_{N-2}-34 y_{N-1}+8 y_{N}=-h^{2} y_{N}^{\prime \prime} \\
& +2 h^{3} y_{N}^{\prime \prime}+\frac{59}{12} h^{4} y_{N-2}^{(4)}+\left(-\frac{5}{3} h^{5} y_{N}^{(5)}+\ldots\right) \text {, } \\
& \text { (e) }-y_{N}-4+12 y_{N}-3-34 y_{N}-2+36 y_{N}-1-13 y_{N}=-4 h^{2} y_{N}^{\prime} \\
& -4 h^{3} y_{N}^{\prime \prime}+\frac{26}{3} h^{4} y_{N-1}^{(4)}+\left(\frac{5}{3} h^{5} y_{N}^{(5)}+\ldots\right) \text {, } \\
& \text { (f) }-y_{N}-3+8 y_{N}-2-13 y_{N-1}+6 y_{N}=5 h^{2} y_{N}^{\prime \prime}-4 y_{N}^{\prime \prime \prime} \\
& +\frac{17}{12} h^{4} y_{N}^{(4)}-\frac{23}{72} h^{6} y^{(6)}\left(\xi_{N}\right), \xi_{N} \in\left(x_{N}-3, b\right) \text {. }
\end{aligned}
$$

This third order method gives rise to a generalized seven-band symmetric matrix eigenvalue problem

$$
\left(B+h^{4} P\right) \tilde{y}=\Lambda h^{4} Q \tilde{y}
$$

where 


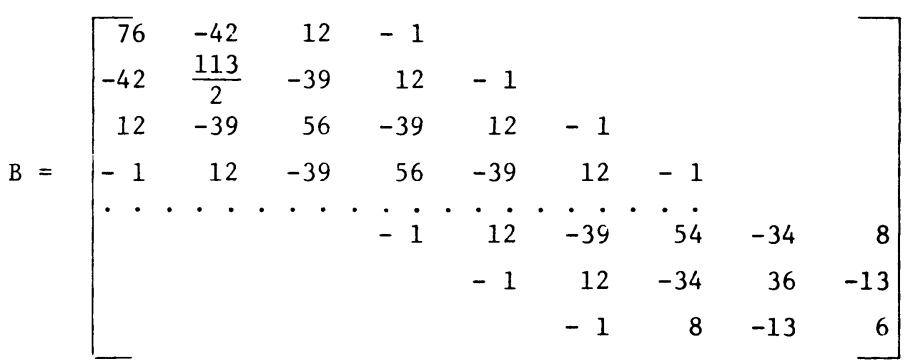

\section{NUEKICAL ILLUSTRATIONS}

To illustrate our methods for order 2 and 4 for the approximation $\lambda$ of (1.1)$(1.2 b)$, re consider the eigenvalue problem

$$
\left\{\begin{array}{l}
y(4)-\frac{\lambda}{(1+x)^{4}} y=0, \\
y(0)=y^{\prime \prime}(0)=y(b)=y^{\prime \prime}(b)=0 .
\end{array}\right.
$$

The smallest eigenvalues $\lambda(b)$ for $b=1,2$ are

$$
\lambda(1)=416.324,564,86 \ldots
$$

and $\lambda(2)=646.269,207, \ldots$ respectively. We computed approximations $\wedge(1)$ and $\wedge(2)$ by our methods (2.1) and (3.1) applied to the problem (5.1) for $\mathrm{h}=2^{-\mathrm{m}}$, $\mathrm{m}=3(1) 6$. The corresponding errors $\left|1-\frac{\lambda(b)}{\wedge(b)}\right|$ are shown in Table I. It is easily verified that our methods based on finite difference approximations (2.1) and (3.1) do provide $O\left(h^{2}\right)$ and $O(h)^{4}$ - convergent approximations for the smallest eigenvalue of $(5.1)$.

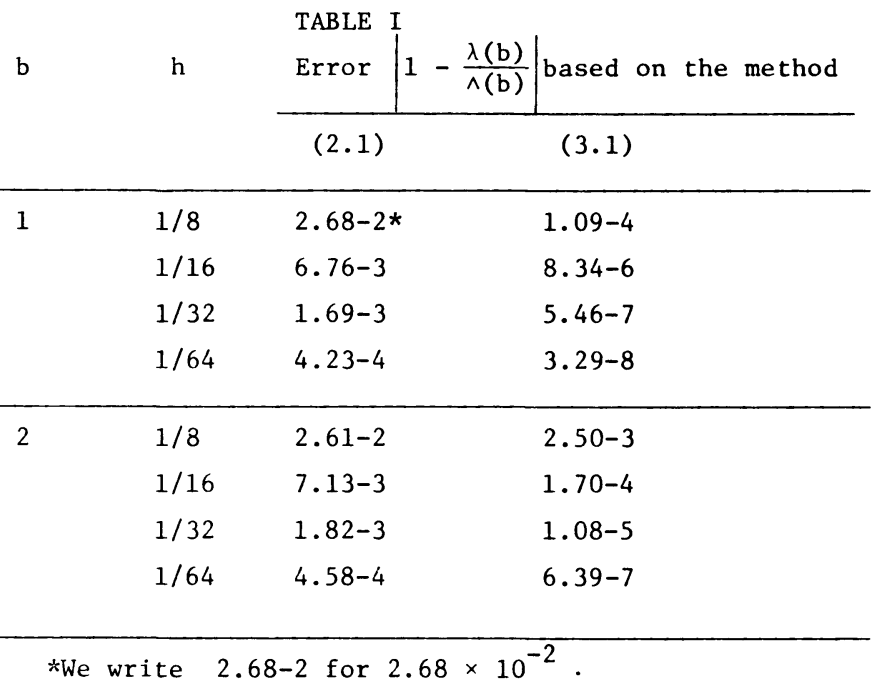

We now illustrate our methods for the approximation of $\lambda$ of $(1.1)-(1.2 c)$ by approximating the value of the smallesteigenvalue $\lambda_{1}$ satisfying

$$
\begin{aligned}
& y^{(4)}+\left(\left(1+x^{2}\right)-\frac{\lambda}{(1+x)^{4}}\right) \quad y=0 \\
& y(0)=y^{\prime}(0)=v^{\prime \prime}(1)=y^{\prime \prime \prime}(1)=0,
\end{aligned}
$$



where $\lambda_{1}=135.320,349,281,57 \ldots$ We 1 ist the errors $\left|1-\frac{\lambda}{\wedge_{1}}\right|_{\text {based on the finite }}$ for $\mathrm{h}=2^{-\mathrm{m}}$,
$\mathrm{m}=3,4,5,6$. It is readily verified that the relative etrors difference scheme $(4.1)$ are $0\left(h^{2}\right)$ - convergent and likewise the relative errors based on the scheme $(4.5)$ are $0\left(h^{3}\right)$ - convergent.

TABLE II

h Error $\left|1-\frac{\lambda_{1}}{\Lambda_{1}}\right|$ based on methods

$(4.1) \quad(4.5)$

\begin{tabular}{lll}
\hline $1 / 8$ & $1.40-2$ & $2.80-3$ \\
$1 / 16$ & $3.41-3$ & $3.50-4$ \\
$1 / 32$ & $8.41-4$ & $4.31-5$ \\
$1 / 64$ & $2.08-4$ & $4.78-6$ \\
$1 / 128$ & $5.13-5$ & \\
\hline
\end{tabular}

It is well known that the tridiagonal matrix $\mathrm{J}$ introduced in (2.4) is a positive definite matrix. It follows that the matrices $\mathrm{J}^{2}$ and $\left(6 \mathrm{~J}^{2}+\mathrm{J}^{3}\right)$ introduced in equations (2.6) and (3.2) respectively are also positive definite matrices. In order to establish that the real symmetric matrix $A$ given by (4.4) is a positive definite matrix, it suffices to prove that the $(\mathrm{N}-1)$ principal minors

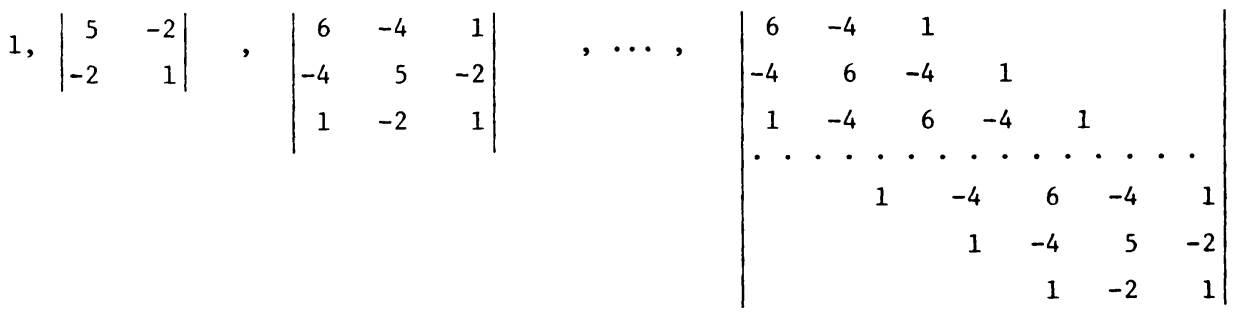

are each equal to 1 and $|A|=2$

ACKNOWLEDGEMENT.

This work was supported in part by a grant from the Natural Sciences and Engineering Research Council of Canada. We also acknowledge the assistance of Mr. Naiyer A. Usmani for making numerical calculations presented in Tables I and II.

\section{REFERENCES}

1. FOX, L. The numerical solution of two-point boundary value problems in ordinary differential equation, Oxford University Press, Oxford, 1957.

2. HILDEBRAND, F.B. Advance Calculus for Applications, Prentice-Hall Inc., Inglewood Cliffs, N.J., 1964 .

3. REISS, E.L. CALLEGARI, A.J. and AHLUWALIA, D.S. Ordinary Differential Equations with Applicatins, Holt, Rinhart and Winston, New York, 1976.

4. CHAWLA, M.M. and KATTI, C.P. A new symmetric five-diagonal finite difference method for computing eigenvalues of fourth-order two-point boundary value problem, J. Comput. and App1. Math., 8 (1982), 135-136. 
5. CHAWLA, M.M. A new fourth-order finite difference method for computing eigenvalues of fourth-order two-point boundary value problem, IMA Journal of Numerical Analysis, $\underline{3}$ (1983), 291-293.

6. GRIGORIEFF, R.D. Diskrete Approximation von Eigenwertproblemen II, Number. Math., 24 (1975), 415-433.

7. USMANI, R.A. On a class of numerical integration methods for a problem of plate deflection theory, Internat. J. Comput. Math., 11 (1982), 305-318.

8. USMANI, R.A. Finite difference methods for a certain two point boundary value problem, Indian J. Pure Appl. Math., 14 (1983), 398-411. 


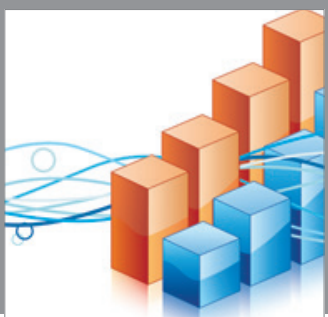

Advances in

Operations Research

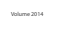

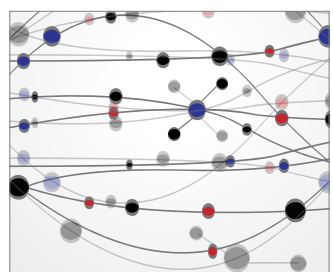

\section{The Scientific} World Journal
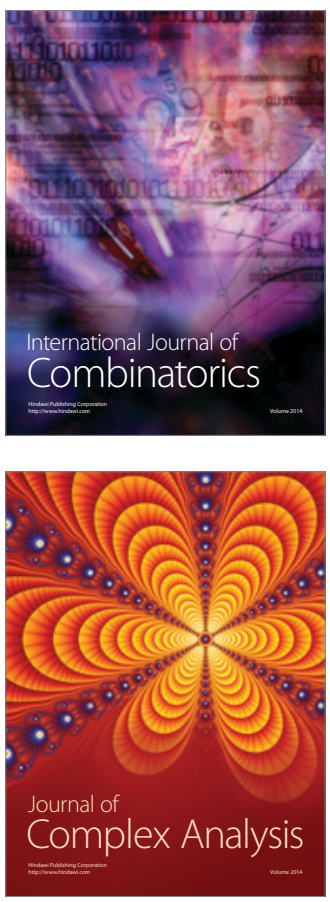

International Journal of

Mathematics and

Mathematical

Sciences
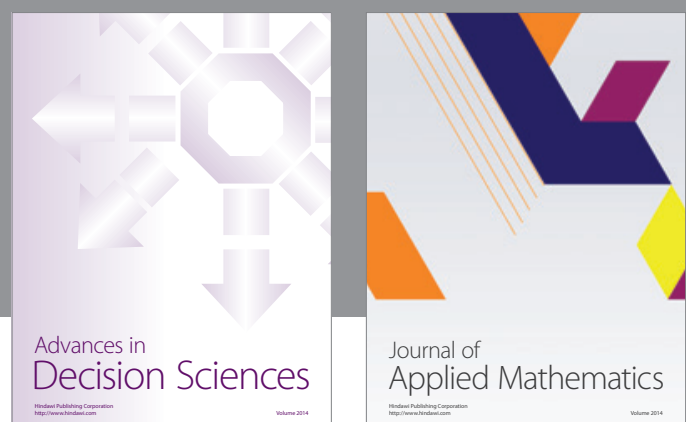

Journal of

Applied Mathematics
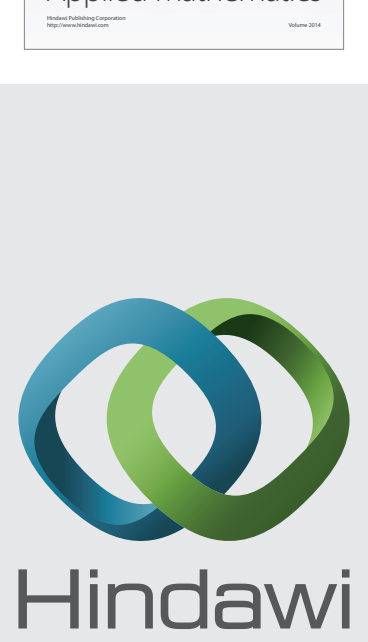

Submit your manuscripts at http://www.hindawi.com
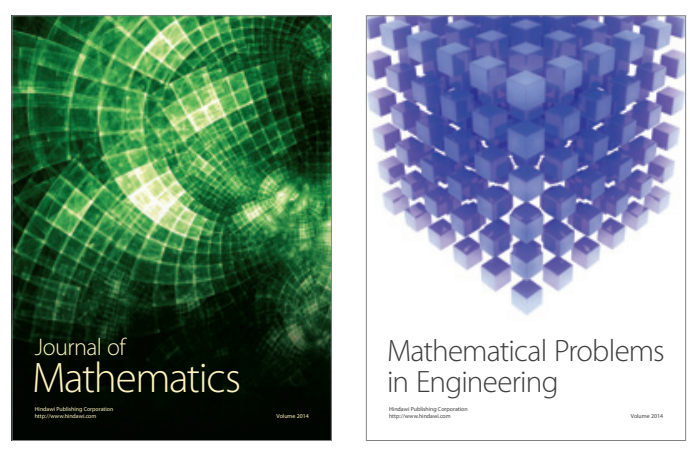

Mathematical Problems in Engineering
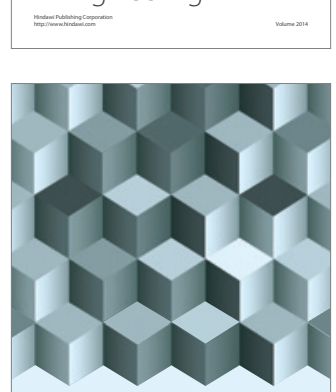

Journal of

Function Spaces
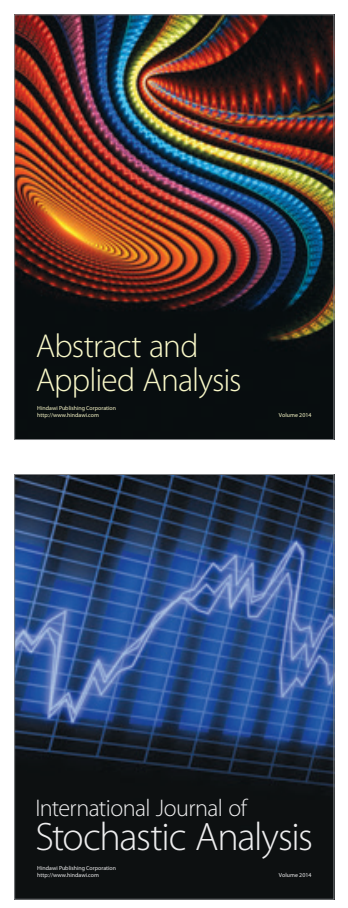

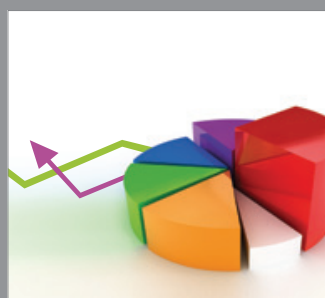

ournal of

Probability and Statistics

Promensencen
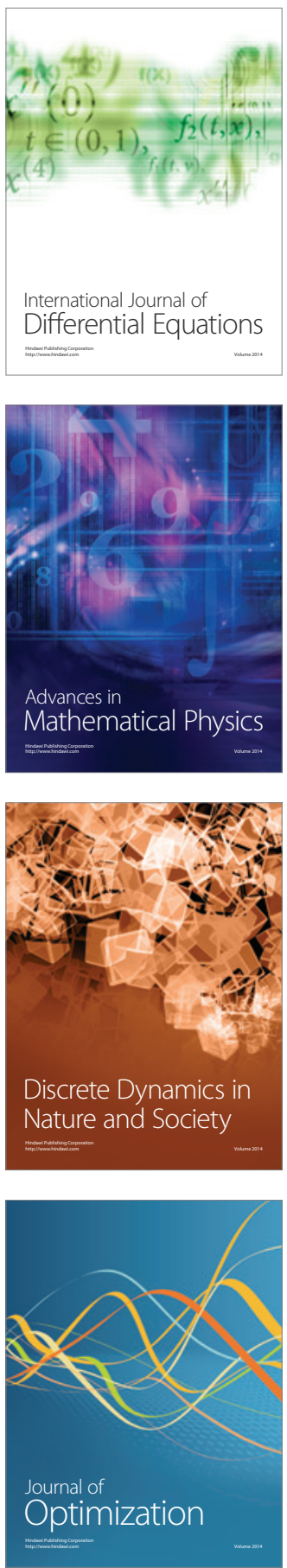